Original

\title{
A Model of Stress Relaxation for Brain Retraction Simulation
}

\author{
Xiaoshuai Chen (Student member $)^{\mathrm{a}}$, Atsushi Konno (Member) ${ }^{\mathrm{a} *}$, Kazuya Sase (Member) ${ }^{\mathrm{b}}$, Akito Ema \\ (Student member) ${ }^{a}$, Teppei Tsujita ${ }^{c}$ \\ ${ }^{a}$ Division of Systems Science and Informatics, Graduate School of Information Science and Technology, \\ Hokkaido University \\ ${ }^{\mathrm{b}}$ Department of Mechanical Engineering and Intelligent Systems, Faculty of Engineering, Tohoku Gakuin \\ University \\ ${ }^{c}$ Department of Mechanical Engineering, National Defense Academy
}

\begin{abstract}
Brain retraction is an important surgical technique that is used for lesion exposure in brain surgeries. However, in brain retraction, an excessive load may cause permanent damage to the brain. Therefore, the development of surgical simulators that can simulate the stress and force of brain reaction in advance of a real surgery is required.

In order to simulate brain retraction, a numerical calculation model of a viscoelastic body using the finite element method (FEM) was proposed. It is based on the generalized Maxwell model but considers the inertial term. The main advantage of including the inertial term is computational stability. The viscoelastic parameters were identified by the results of retraction experiments using whole porcine brains. Retraction experiments were performed with two retraction velocities, $1 \mathrm{~mm} / \mathrm{s}$ and $10 \mathrm{~mm} / \mathrm{s}$. The parameters of the proposed model were identified by solving optimization problems using the retraction experimental results of the retraction velocity $10 \mathrm{~mm} / \mathrm{s}$. Retraction simulations (retraction velocity :1 mm/s and $10 \mathrm{~mm} / \mathrm{s}$ ) were performed under the same conditions as in the experiments. The 3D simulation model was built from MRI data of a porcine brain. Comparing the simulation results and experimental data, it is confirmed that the viscoelastic model using the identified parameters simulated the brain retraction well.
\end{abstract}

\section{Key words}

brain surgery simulation, brain retraction, viscoelasticity, FEM, retraction experiment

\section{Introduction}

In the medical field, the demand for surgery simulators that use virtual reality technology has been increasing rapidly. Because the models in the simulation can be made from medical images of a real patient and there is no risk of injuery to the patient, surgery simulators are required especially in the following situations :

- Surgery simulators offer opportunities of training for novice surgeons ${ }^{1,2)}$.

\footnotetext{
*Division of System Science and Informatics, Graduate School of Information Science and Technology, Hokkaido University, Kita 14, Nishi 9, Kita-ku, Sapporo, Hokkaido, 060-0814, Japan konno@ssi.ist.hokudai.ac.jp Received 2016/9/9 Accepted 2017/5/8
}

- Surgeons can create and verify a preoperative plan by performing a simulation.

- Surgeons can simulate the surgery with realistic 3D organ models of patients. Models are made from CT or MRI images. This is especially useful for cases involving rare diseases.

In response to the demand, several neurosurgery simulators have been developed so far, such as ImmersiveTouch $^{3)}$ and NeuroTouch ${ }^{4)}$. The usefulness of neurosurgery simulators has been confirmed ${ }^{5), 6)}$.

Brain retraction is a fundamental technique in neurosurgeries. It is used to obtain sufficient exposure to create a path to a tumor or an aneurysm. Previous studies have reported that excessive stress at brain retraction is 


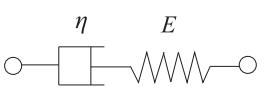

(a)

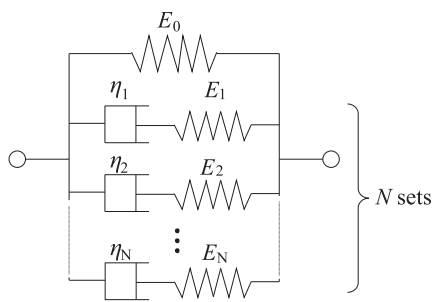

(b)
Fig. 1 Schematic of (a) Maxwell model and (b) generalized Maxwell model.

deleterious because it may cause brain injury such as contusion, infarction, edema and so $\mathrm{on}^{7)-10)}$. In order to establish a serviceable brain surgery simulation, a function to warn neurosurgeons about potential brain retraction injury should be incorporated in the simulation. The brain retraction injury is caused by the pressure of the retractors and its duration ${ }^{7)}$. Therefore, an accurate presentation of stress distribution in brain tissues is required.

Several previous studies have reported the mechanical characteristic of the brain (human ${ }^{11)}$, swine ${ }^{12)}$, bovine ${ }^{13)}$ ). These studies described a phenomenon called stress relaxation, that is generally seen in brain retraction. Stress relaxation is usually described by viscoelasticity using the generalized Maxwell model ${ }^{14)}$, which comprises a spring and $N$ sets of the Maxwell model (Fig. 1).

Several earlier studies have reported the analysis results of brain tissue obtained with the generalized Maxwell model ${ }^{12,13,15)}$. Most of them did not consider an inertial term. In a surgical simulation, surgical cuts and dissection must be simulated. However, when a brain finite element (FE) model is cut, isolated elements or structurally unstable elements are easily generated and such elements make the stiffness matrix singular. The displacement of nodes is generally calculated by inversion of the stiffness matrix, and hence if the stiffness matrix becomes singular, the computation of the displacement of nodes becomes unstable. However, by considering an inertial term, the computation is always feasible. Therefore, the inertial term should be included. Moreover, in most earlier studies, the mechanical characteristics of the brain were tested with a part of the brain. However, such tests cannot correctly measure the stress generation, which depends on the structure of the entire brain.

In this study, a viscoelastic model is presented, that is based upon the generalized Maxwell model ; however an inertial term is included. Experiments were conducted with whole porcine brains. The parameters were identified by solving an optimization problem so that the simulation with a porcine brain model reproduces the experimental results well.

\section{Numerics of Viscoelasticity}

\subsection{Numerical analysis of generalized Maxwell model using FEM $^{16), 17)}$}

The finite element method (FEM) is used as a numerical analysis technique for three-dimensional generalized Maxwell model. In a one-dimensional model, the stress $\sigma$ at time $t$ of the generalized Maxwell model is given by

$$
\sigma(t)=\int_{0}^{t} E(t-s) \frac{\partial \varepsilon}{\partial s} d s
$$

where

$$
\begin{aligned}
& E(t)=E_{0}+\sum_{i=1}^{N} E_{i} \exp \left(-\frac{t}{\tau_{i}}\right), \\
& \tau_{i}=\frac{\eta_{i}}{E_{i}},
\end{aligned}
$$

$s$ is the starting time and $\varepsilon$ is the strain. $\tau_{i}$ is called the relaxation time. Splitting $\sigma(t)$ into an elastic term and a viscoelastic term, (1) becomes

$$
\begin{aligned}
\sigma(t) & =E_{0} \varepsilon(t)+\sum_{i=1}^{N} \int_{0}^{t} E_{i} \exp \left(-\frac{t-s}{\tau_{i}}\right) \frac{\partial \varepsilon}{\partial s} d s \\
& =\sigma_{0}(t)+\sum_{i=1}^{N} h_{i}(t) .
\end{aligned}
$$

When the strain $\varepsilon$ is constant, $\lim _{t \rightarrow 0} h_{i}(t)=0$, and $\lim _{t \rightarrow 0} \sigma(t)$ $=\sigma_{0}$.

(4) is discretized and extended to the three-dimensional model as follows :

$$
\boldsymbol{\sigma}^{(n+1)}=\boldsymbol{\sigma}_{0}^{(n+1)}+\sum_{i=1}^{N} \mathbf{h}_{i}^{(n+1)},
$$

where

$$
\mathbf{h}_{i}^{(n+1)}=\exp \left(-\frac{\Delta t}{\tau_{i}}\right) \mathbf{h}_{i}^{(n)}+r_{i} A_{i}\left[\boldsymbol{\sigma}_{0}^{(n+1)}-\boldsymbol{\sigma}_{0}^{(n)}\right],
$$




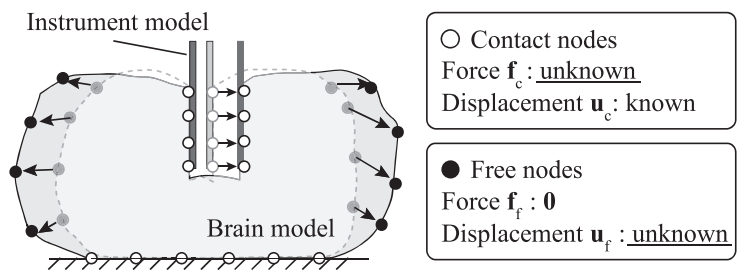

Fig. 2 Knowns and unknowns of contact nodes and free nodes.

$$
\begin{aligned}
& A_{i}=\frac{1-\exp \left(-\Delta t / \tau_{i}\right)}{\Delta t / \tau_{i}}, \\
& r_{i}=E_{i} / E_{0} .
\end{aligned}
$$

In FEM, the elastic term $\boldsymbol{\sigma}_{0}^{(n+1)}$ of an element is calculated by

$$
\left(\boldsymbol{\sigma}_{\mathrm{e}}\right)_{0}^{(n+1)}=\mathbf{D}_{\mathrm{e}} \mathbf{B}_{\mathrm{e}} \mathbf{u}_{\mathrm{e}}^{(n+1)},
$$

where $\mathbf{B}$ is a constant matrix defined by the shape functions, $\mathbf{D}$ is a symmetric material stiffness matrix, and $\mathbf{u}$ is the displacement vector of the nodes. The subscript "e" denotes that the matrix or vector is of an element.

Substituting (9) into (5) and (6), the stress vector is given by

$$
\begin{aligned}
\boldsymbol{\sigma}_{\mathrm{e}}^{(n+1)}= & \left(1+\sum_{i=1}^{N} r_{i} A_{i}\right) \mathbf{D}_{\mathrm{e}} \mathbf{B}_{\mathrm{e}} \mathbf{u}_{\mathrm{e}}^{(n+1)} \\
& +\sum_{i=1}^{N} \exp \left(-\frac{\Delta t}{\tau_{i}}\right) \mathbf{h}_{i}^{(n)}-\left(\sum_{i=1}^{N} r_{i} A_{i}\right) \mathbf{D}_{\mathrm{e}} \mathbf{B}_{\mathrm{e}} \mathbf{u}_{\mathrm{e}}^{(n)}, \\
\mathbf{h}_{i}^{(n+1)}= & \exp \left(-\frac{\Delta t}{\tau_{i}}\right) \mathbf{h}_{i}^{(n)}+r_{i} A_{i} \mathbf{D}_{\mathrm{e}} \mathbf{B}_{\mathrm{e}}\left(\mathbf{u}_{\mathrm{e}}^{(n+1)}-\mathbf{u}_{\mathrm{e}}^{(n)}\right)
\end{aligned}
$$

The force vector $\mathbf{f}$ is formulated as

$$
\mathbf{f}^{(n+1)}=\mathbf{K}_{\mathrm{n}} \mathbf{u}^{(n+1)}+\mathbf{f}_{\text {hist }}^{(n+1)}
$$

where

$$
\begin{aligned}
& \mathbf{K}_{\mathrm{n}}=\underset{\mathrm{e}=1}{\mathrm{all}}\left(\mathbf{K}_{\mathrm{e}}\right)_{\mathrm{n}}, \\
& \left(\mathbf{K}_{\mathrm{e}}\right)_{\mathrm{n}}=\left(1+\sum_{i=1}^{N} r_{i} A_{i}\right) \mathbf{K}_{\mathrm{e}}, \\
& \mathbf{K}_{\mathrm{e}}=\int_{V_{\mathrm{e}}} \mathbf{B}_{\mathrm{e}}^{\mathrm{T}} \mathbf{D}_{\mathrm{e}} \mathbf{B}_{\mathrm{e}} d V,
\end{aligned}
$$

$$
\begin{aligned}
& \mathbf{f}_{\text {hist }}=\underset{\mathrm{e}=1}{\mathbf{a l l}}\left(\mathbf{f}_{\mathrm{e}}\right)_{\text {hist, }} \\
& \left(\mathbf{f}_{\mathrm{e}}\right)_{\text {hist }}^{(n+1)}=\mathbf{h}_{\text {hist }}^{(n+1)}-\left(\mathbf{K}_{\mathrm{e}}\right)_{\text {hist }} \mathbf{u}_{\mathrm{e}}^{(n)}, \\
& \mathbf{h}_{\text {hist }}^{(n+1)}=\left(V_{\mathrm{e}} \mathbf{B}_{\mathrm{e}}^{\mathrm{T}}\right) \sum_{i=1}^{N} \exp \left(-\frac{\Delta t}{\tau_{i}}\right) \mathbf{h}_{i}^{(n)}, \\
& \left(\mathbf{K}_{\mathrm{e}}\right)_{\text {hist }}=\left(\sum_{i=1}^{N} r_{i} A_{i}\right) \mathbf{K}_{\mathrm{e},}
\end{aligned}
$$

$\mathbf{A}_{\mathrm{e}=1}^{\text {all }}$ is a finite element assembly operator, and $V_{\mathrm{e}}$ is the volume of the element.

\subsection{A numerical model of viscoelasticity with an inertial term}

The FEM implementation of the generalized Maxwell model was formulated as $(12)^{16), 17)}$. Equation (12) does not include an inertial term. As mentioned in Introduction, the inertial term makes the computation always feasible. Therefore, a viscoelastic model with an inertial term is proposed as follows :

$$
\mathbf{f}^{(n+1)}=\mathbf{K}_{\mathrm{n}} \mathbf{u}^{(n+1)}+\mathbf{f}_{\text {hist }}^{(n+1)}+\mathbf{M} \ddot{\mathbf{u}}^{(n+1)},
$$

where $\mathbf{M}$ is the mass matrix and $\ddot{\mathbf{u}}$ is the acceleration vector of the nodes. It is assumed that the masses are lumped at the nodes, and hence $\mathbf{M}$ becomes diagonal. The $i$ th diagonal block matrix $\mathbf{M}_{i}$ is $m_{i} \mathbf{I}_{3}$, where $m_{i}$ is the lumped mass of node $i$ and $\mathbf{I}_{3}\left(\in \mathrm{R}^{3 \times 3}\right)$ is an identity matrix.

$\ddot{\mathbf{u}}$ is updated by the constant average acceleration method $^{18)}$, which is an implicit method that guarantees unconditional stability in numerical computation. The update rule is as follows :

$$
\begin{aligned}
& \ddot{\mathbf{u}}^{(n+1)}=\frac{4}{\Delta t^{2}}\left(\mathbf{u}^{(n+1)}-\mathbf{u}^{(n)}\right)-\frac{4}{\Delta t} \dot{\mathbf{u}}^{(n)}-\ddot{\mathbf{u}}^{(n)}, \\
& \dot{\mathbf{u}}^{(n+1)}=\frac{2}{\Delta t}\left(\mathbf{u}^{(n+1)}-\mathbf{u}^{(n)}\right)-\dot{\mathbf{u}}^{(n)} .
\end{aligned}
$$

In this study, the nodes that contact with surgical instruments or the stage are assumed to move together with the surgical instruments and the stage. Such nodes are called contact nodes ( $\bigcirc$ " in Fig. 2) in this paper. The nodes that do not contact with surgical instruments are 


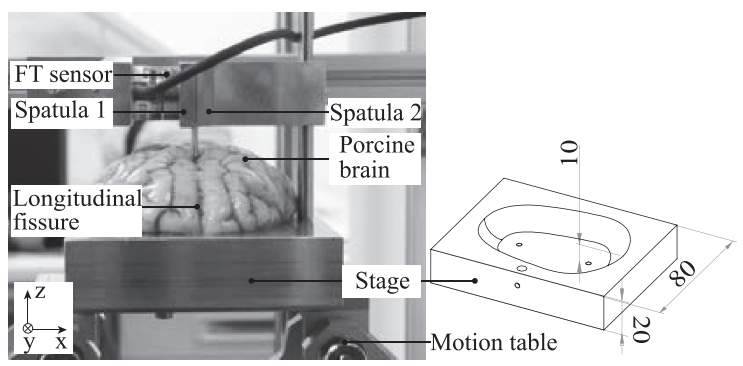

Fig. 3 Experimental equipment for splitting brain fissure.

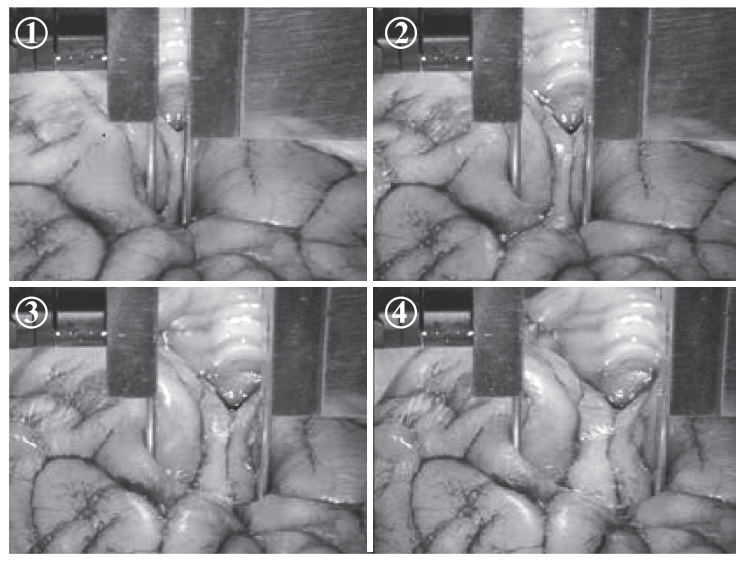

Fig. 4 Sequences of an experiment (specimen No. 11). The retraction velocity is $1 \mathrm{~mm} / \mathrm{s}$.

called free nodes ("O" in Fig. 2). In (20), the unknowns of contact nodes and free nodes are different. For contact nodes, the displacement vectors are known, whereas the force vectors are unknown. In contrast, for free nodes, the force vectors are $\mathbf{0}$, whereas the displacement vectors are unknown. Therefore, the force and displacement for contact nodes and free nodes are calculated separately, and (20) is divided as follows :

$$
\begin{aligned}
{\left[\begin{array}{l}
\mathbf{f}_{\mathrm{c}}^{(n+1)} \\
\mathbf{f}_{\mathrm{f}}^{(n+1)}
\end{array}\right]=} & {\left[\begin{array}{ll}
\left(\mathbf{K}_{\mathrm{cc}}\right)_{\mathrm{n}} & \left(\mathbf{K}_{\mathrm{cf}}\right)_{\mathrm{n}} \\
\left(\mathbf{K}_{\mathrm{fc}}\right)_{\mathrm{n}} & \left(\mathbf{K}_{\mathrm{ff}}\right)_{\mathrm{n}}
\end{array}\right]\left[\begin{array}{l}
\mathbf{u}_{\mathrm{c}}^{(n+1)} \\
\mathbf{u}_{\mathrm{f}}^{(n+1)}
\end{array}\right] } \\
& +\left[\begin{array}{c}
\left(\mathbf{f}_{\mathrm{c}}\right)_{\text {hist }}^{(n+1)} \\
\left(\mathbf{f}_{\mathrm{f}}\right)_{\text {hist }}^{(n+1)}
\end{array}\right]+\left[\begin{array}{cc}
\mathbf{M}_{\mathrm{c}} & \mathbf{0} \\
\mathbf{0} & \mathbf{M}_{\mathrm{f}}
\end{array}\right]\left[\begin{array}{l}
\ddot{\mathbf{u}}_{\mathrm{c}}^{(n+1)} \\
\ddot{\mathbf{u}}_{\mathrm{f}}^{(n+1)}
\end{array}\right],
\end{aligned}
$$

where the subscript "c" denotes the contact node related terms, and the subscript " $\mathrm{f}$ " denotes the free node related terms.

Because the contact nodes move with the surgical instrument operated by a surgeon, $\mathbf{u}_{\mathrm{c}}^{(n+1)}$ is known, while $\mathbf{f}_{\mathrm{f}}^{(n+1)}=\mathbf{0}$. Substituting (21) into (23), the displacement of the free nodes $\mathbf{u}_{\mathrm{f}}^{(n+1)}$ and the external force of the contact nodes $\mathbf{f}_{\mathrm{c}}^{(n+1)}$ are calculated separately as follows :

$$
\begin{aligned}
\mathbf{u}_{\mathrm{f}}^{(n+1)}= & -\left[\left(\mathbf{K}_{\mathrm{ff}}\right)_{\mathrm{n}}+\frac{4}{\Delta t^{2}} \mathbf{M}_{\mathrm{f}}\right]^{-1}\left[\left(\mathbf{K}_{\mathrm{fc}}\right)_{\mathrm{n}} \mathbf{u}_{\mathrm{c}}^{(n+1)}\right. \\
& +\left(\mathbf{f}_{\mathrm{f}}\right)_{\text {hist }}^{(n+1)}-\mathbf{M}_{\mathrm{f}}\left(\frac{4}{\Delta t^{2}} \mathbf{u}_{\mathrm{f}}^{(n)}+\frac{4}{\Delta t} \dot{\mathbf{u}}_{\mathrm{f}}^{(n)}\right. \\
& \left.\left.+\ddot{\mathbf{u}}_{\mathrm{f}}^{(n+1)}\right)\right],
\end{aligned}
$$

$$
\begin{aligned}
\mathbf{f}_{\mathrm{c}}^{(n+1)}= & \left(\left(\mathbf{K}_{\mathrm{cc}}\right)_{\mathrm{n}}+\frac{4}{\Delta t^{2}} \mathbf{M}_{\mathrm{c}}\right) \mathbf{u}_{\mathrm{c}}^{(n+1)}+\left(\mathbf{K}_{\mathrm{fc}}\right)_{\mathrm{n}} \mathbf{u}_{\mathrm{f}}^{(n+1)} \\
& +\left(\mathbf{f}_{\mathrm{c}}\right)_{{ }_{\text {hist }}^{(n+1)}}^{(n+} \mathbf{M}_{\mathrm{c}}\left(\frac{4}{\Delta t^{2}} \mathbf{u}_{\mathrm{c}}^{(n)}+\frac{4}{\Delta t} \dot{\mathbf{u}}_{\mathrm{c}}^{(n)}\right. \\
& \left.+\ddot{\mathbf{u}}_{\mathrm{c}}^{(n+1)}\right) .
\end{aligned}
$$

The reaction force of an area is calculated by the sum of the forces of the nodes in the area :

$$
\mathbf{f}_{\mathrm{re}}=\sum_{i=1}^{N_{\mathrm{c}}} \mathbf{f}_{i}
$$

where $N_{\mathrm{c}}$ is the number of nodes in the area.

The displacement vector $\mathbf{u}_{\mathrm{f}}^{(n+1)}$ of the free nodes is calculated by (24). It is easily understood that if the mass matrix $\mathbf{M}_{\mathrm{f}}$ is not included, the calculation (24) becomes unstable when $\left(\mathbf{K}_{\mathrm{ff}}\right)_{\mathrm{n}}$ is approximately singular. By considering the inertial term with a diagonal mass matrix $\mathbf{M}$, the calculation (24) is always feasible and stable. This is the main advantage of including the inertial term.

\section{Retraction Experiments at Longitudinal Fissure of Cerebrum}

In order to use a brain that is as close to the living state as possible, porcine heads were collected from a local slaughterhouse on the morning of the experiment day. Whole brain tissues except the dura mater were enucleated from the heads. Brains were stored in saline at room temperature.

The experimental system is shown in Fig. 3. As shown in the figure, the bottom shape of the brain was carved in the stage. The brain was directly placed on it. Two spatulas (Spatula1 and Spatula2) were inserted in the middle of the longitudinal cerebral fissure. The width of the spatulas was $5 \mathrm{~mm}$, and the inserted depth was $15 \mathrm{~mm}$. Spatulal was fixed to a $6^{-}$axis force sensor (NANO 17, ATI Industrial 


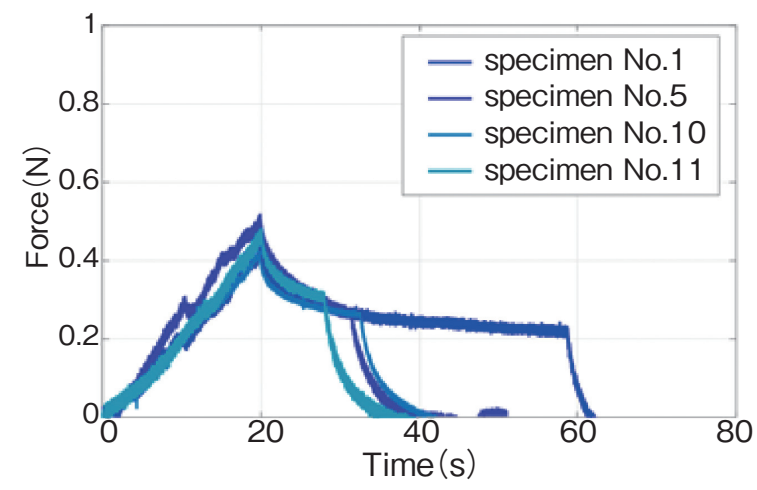

Fig. 5 Retraction force results of experiments (four specimens) with retraction velocity of $1 \mathrm{~mm} / \mathrm{s}$.

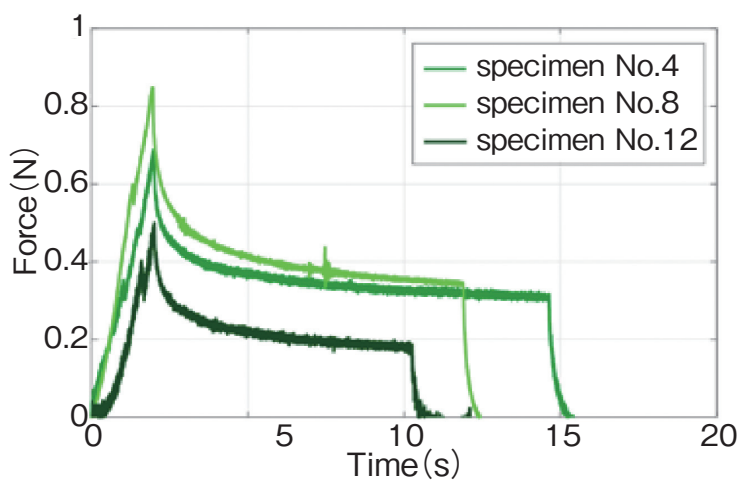

Fig. 6 Retraction force results of experiments (three specimens) with retraction velocity of $10 \mathrm{~mm} / \mathrm{s}$.

Automation, Inc.), and Spatula2 was fixed to the stage, which was attached to the motion table, and moved in the positive $\mathrm{x}^{-}$axis direction. Therefore, Spatula1 provided a forced displacement in the negative $\mathrm{x}$-axis direction to the brain.

In the experiments, the motion table moved $20 \mathrm{~mm}$ at 1 $\mathrm{mm} / \mathrm{s}$ and $10 \mathrm{~mm} / \mathrm{s}$, remained stationary to maintain the deformation for several seconds, and then returned to the initial position. Fig. 4 shows the sequences of the retraction experiment. Twelve porcine brains were used in the retraction experiments. Among the 12 experiments, 2 were unsuccessful in acquiring force data. Three experimental results deviated greatly from the mean values, and hence they were not used for parameter identification. The force generated at Spatula1 along the $\mathrm{x}^{-}$axis in the other seven retraction experiments are plotted in Fig. 5 and 6. Specimens Nos. 1, 5, 10, and 11 were retracted at $1 \mathrm{~mm} / \mathrm{s}$, while specimens Nos. 4, 8, and 12 were retracted at 10 $\mathrm{mm} / \mathrm{s}$. The stationary times of the motion table after retraction were varied in each experiment.

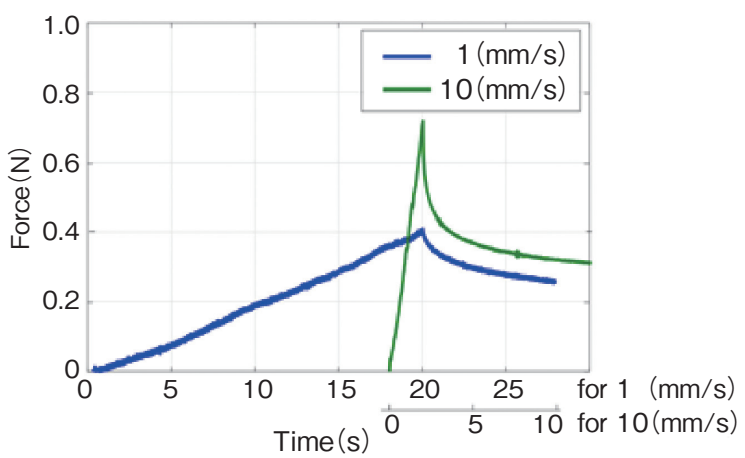

Fig. 7 Average of experimental results of force with retraction velocities of $1 \mathrm{~mm} / \mathrm{s}$ and $10 \mathrm{~mm} / \mathrm{s}$.

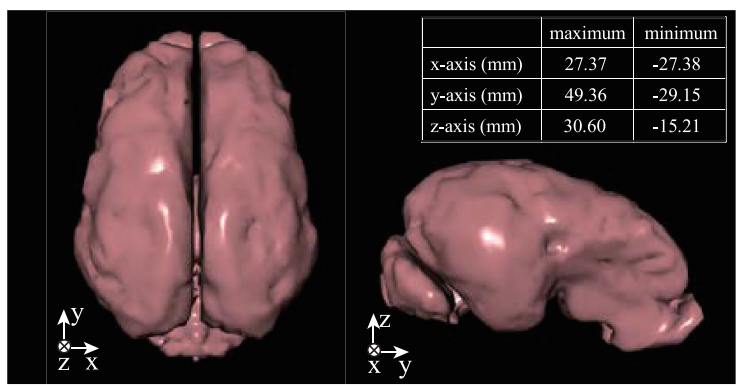

Fig. 8 The porcine brain FE model transformed from the atlas data $^{19), 20)}$.

As seen in Fig. 6, sudden decreases in the retraction force are seen when the brain is retracted (e.g. the result of specimen No. 12 at $1.6 \mathrm{~s}$ ). It appears that when connective tissues such as the arachnoid trabeculae and the blood vessels filling the brain fissure are fractured, the retraction force decreases. Because the decreases in the retraction force of specimen No. 12 at approximately $1.6 \mathrm{~s}$ greatly affected the peak value of the force, the result of specimen No. 12 was not used for parameter identification. In the retraction experiments with a retraction velocity of $1 \mathrm{~mm} /$ $\mathrm{s}$, the connective tissues were gradually fractured, and hence the fracture did not seriously affect the retraction force, as shown in Fig. 5.

For comparison, experimental data were averaged at each moving speed. The averages of the experimental results with a retraction velocity of $1 \mathrm{~mm} / \mathrm{s}$ and $10 \mathrm{~mm} / \mathrm{s}$ are plotted in Fig. 7. In order to compare the stress relaxation, the time axis for the experimental result with a velocity of $10 \mathrm{~mm} / \mathrm{s}$ is shifted so that the times of the force peaks meet (see the time axes of Fig. 7). Because the stationary times of the motion table were different in each 
experiment, only 0 to $27.95 \mathrm{~s}$ of $1 \mathrm{~mm} / \mathrm{s}$ data and $12 \mathrm{~s}$ of $10 \mathrm{~mm} / \mathrm{s}$ data were used in the calculation of averages. As shown in Fig. 7, the strain rate dependency is reflected.

\section{Retraction Simulation}

\subsection{Simulation scenario}

The sequences of the retraction experiments were reproduced as faithfully as possible in the simulations with the following settings.

\subsubsection{Simulation model}

Saikali constructed a digital atlas ${ }^{19)}$ of a porcine brain from MRI images, which were taken from a six-monthold female pig. The atlas data are available from 20). In this study, the porcine heads were from a slaughterhouse, where the pigs were almost six months old. The size and weight of the brains in 19) were similar to the specimens used in the experiments. Therefore, the brain model is suitable for the simulation. In the experiments, when the fissure was retracted, some connective tissues and membrane were dissected. However, the brain model only contains cerebral parenchyma. The effect of this difference was hypothesized to be insignificant.

The porcine brain atlas data ${ }^{20)}$ were transformed into a FE model that was composed of 5,212 nodes and 25,102 tetrahedral elements. The internal cavities were plugged, and fragments separated from the main body of brain tissue were eliminated. The brain is considered to be homogeneous. The size and shape of the porcine brain model are shown in Fig. 8.

\subsubsection{Contact nodes}

In the experiments, two spatulas were inserted into the longitudinal cerebral fissure. The width of a spatula was 5 $\mathrm{mm}$, and the insertion depth was $15 \mathrm{~mm}$. The surface nodes of the brain model that contacted with the spatula were classified as contact nodes. Moreover, the bottom of the brain moved with the motion table, and therefore the surface nodes in this part were also classified as contact nodes

Let $\mathbf{p}_{j}=\left[x_{j} y_{j} z_{j}\right]^{\mathrm{T}}(\mathrm{mm})$ be a position of node $j$. The nodes touching Spatula1 and Spatula2 are denoted by the subscripts “( $(\mathrm{sp} 1)$ " and “( $(\mathrm{sp} 2)$," respectively. It is assumed that the surface nodes in the area :

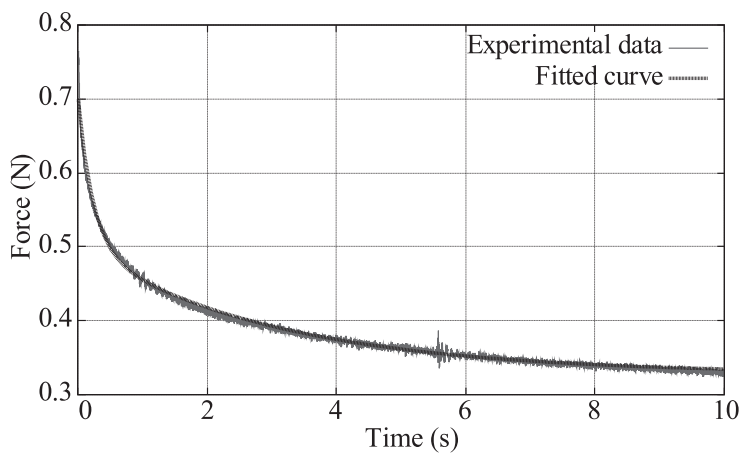

Fig. 9 Curve fitting $(N=2)$ to average experimental results of force with retraction velocity of $10 \mathrm{~mm} / \mathrm{s} . f_{\mathrm{sp} 1}(t)=0.3282+$ $0.1727 \exp (-t / 3.0430)+0.1986 \exp (-t / 0.2312)$

$$
\begin{aligned}
& x_{j} \in\left[\begin{array}{ll}
-1, & 0
\end{array}\right], \quad y_{j} \in\left[\begin{array}{ll}
15, & 20
\end{array}\right], \\
& z_{j} \in\left[z_{\max (\mathrm{sp} 1)}-15, \quad z_{\max (\mathrm{sp} 1)}\right]
\end{aligned}
$$

contact with Spatula1, where $z_{\max }$ is the maximum $\mathrm{z}^{-}$axis position of the nodes in this area.

Similarly, it is assumed that the surface nodes in the area :

$$
\begin{aligned}
& x_{j} \in\left[\begin{array}{ll}
0, & 1
\end{array}\right], \quad y_{j} \in\left[\begin{array}{ll}
15, & 20
\end{array}\right], \\
& z_{j} \in\left[z_{\max (\mathrm{sp} 2)}-15, \quad z_{\max (\mathrm{sp} 2)}\right]
\end{aligned}
$$

contact with Spatula2.

As shown in Fig. 3, the depth of the carved part of the stage is $10 \mathrm{~mm}$. The minimum $\mathrm{z}^{-}$axis position of the nodes of the brain model is $-15.04 \mathrm{~mm}$. Therefore, the surface nodes in the area

$$
z_{j} \in[-15.04,-5.04]
$$

are assumed to be fixed nodes

\subsubsection{Forced displacement of contact nodes}

In the experiments, Spatula2 and the stage were moved, while Spatula1 was fixed (see Fig. 3). Hence, in the simulations, the nodes that contacted with virtual Spatula1 were fixed $(\mathbf{u}=\mathbf{0})$, and the number of those nodes was counted as $N_{\mathrm{c}}$ in (26) to calculate the reaction force. The nodes that contacted with the virtual Spatula2 or the virtual stage were moved in the same way as in the experiments. The final displacement of the movement was $20 \mathrm{~mm}$, and the retraction velocities were $1 \mathrm{~mm} / \mathrm{s}$ and $10 \mathrm{~mm} / \mathrm{s}$. 


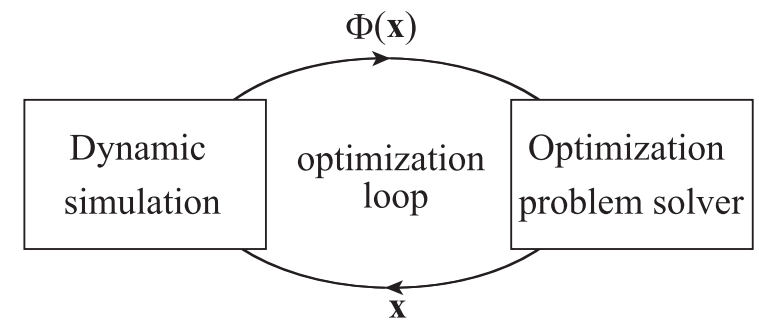

Fig. 10 Identification process.

\subsection{Parameter identification for the proposed model}

\subsubsection{Curve fitting to force relaxation results}

Let $f_{\mathrm{sp} 1}$ be the retraction force generated at Spatula1 along the $\mathrm{x}$-axis. The stress relaxation region of the experimental force-time curves are fitted using fittype () of MATLAB ${ }^{\circledR}$. The equation of curve fitting is set as follows :

$$
f_{\mathrm{sp} 1}(t)=a+\sum_{i=1}^{N} b_{i} \exp \left(-\frac{t}{c_{i}}\right)
$$

An example of fitting assuming $N=2$ to the average force with a retraction velocity of $10 \mathrm{~mm} / \mathrm{s}$ is plotted in Fig. 9. The determination coefficients $\left(R^{2}\right)$ are $0.949(N=$ 1), $0.996(N=2), 0.999(N=3), 0.999(N=4)$, and $0.996(N=5)$. The fitting accuracy does not vary significantly.

\subsubsection{Identification of $E_{0}$}

First, $E_{0}$ was identified. In the stationary condition, $\ddot{\mathbf{u}}=$ $\mathbf{0}, \dot{\mathbf{u}}=\mathbf{0}$ the force generated at the contact nodes is calculated from (23) as $\mathbf{f}_{\mathrm{c}}=\mathbf{K}_{\mathrm{re}} \mathbf{u}_{\mathrm{c}}\left(\mathbf{K}_{\mathrm{re}}=\mathbf{K}_{\mathrm{cc}}-\mathbf{K}_{\mathrm{cf}} \mathbf{K}_{\mathrm{ff}}^{-1} \mathbf{K}_{\mathrm{fc}}\right)$ when $t \rightarrow \infty \quad$ (note that $\left(\mathbf{K}_{\mathrm{e}}\right)_{\mathrm{n}}=\mathbf{K}_{\mathrm{e}}$ as $\left.t \rightarrow \infty\right) . \mathbf{K}_{\mathrm{cc}}, \mathbf{K}_{\mathrm{cc}}, \mathbf{K}_{\mathrm{fc}}$, and $\mathbf{K}_{\mathrm{ff}}$ are the minor matrices of a stiffness matrix, and they are linear to Young's modulus $E$. Hence $\mathbf{K}_{\mathrm{re}}$ can be rewritten as $\mathbf{K}_{\mathrm{re}}(E)=E \tilde{\mathbf{K}}_{\mathrm{re}}$. Therefore, $\mathbf{f}_{\mathrm{c}}\left(E_{0}, t=\infty\right)$ can be written as follows :

$$
\mathbf{f}_{\mathrm{c}}\left(E_{0}, t=\infty\right)=E_{0} \tilde{\mathbf{K}}_{\mathrm{re}} \mathbf{u}_{\mathrm{c}} .
$$

The force $f_{\mathrm{sp} 1}$ that is generated at Spatula1 along the $\mathrm{x}^{-}$ axis is given by

$$
f_{\mathrm{sp} 1}=\mathbf{g}_{\mathrm{sp} 1}^{\mathrm{T}} \mathbf{f}_{\mathrm{c}},
$$

where $\mathbf{g}_{\text {sp1 }}^{\mathrm{T}}$ is a row vector that transforms $\mathbf{f}_{\mathrm{c}}$ into the force $f_{\text {sp1. }}$. Therefore, $f_{\text {sp1 }}\left(\bar{E}_{0}, t=\infty\right)$ is given as follows :
Table 1 Comparison of identified parameters. I : average of parameters identified for No. 4 and No. $8(N=2)$, II : parameters identified using the average of experimental results $(N=2)$, III : parameters identified in 21) $(N=2)$, and IV : parameters identified using the average of experimental results $(N=1)$.

\begin{tabular}{l|rrrrc}
\hline & \multicolumn{1}{|c}{$\begin{array}{c}E_{0} \\
(\mathrm{~Pa})\end{array}$} & $\begin{array}{c}E_{1} \\
(\mathrm{~Pa})\end{array}$ & \multicolumn{1}{c}{$\begin{array}{c}E_{2} \\
(\mathrm{~Pa})\end{array}$} & \multicolumn{1}{c}{$\begin{array}{c}\tau_{1} \\
(\mathrm{~s})\end{array}$} & \multicolumn{1}{c}{$\begin{array}{c}\tau_{2} \\
(\mathrm{~s})\end{array}$} \\
\hline No.4 & 2939.1 & 1629.1 & 5150.1 & 3.97 & 0.55 \\
No. 8 & 3416.5 & 3429.6 & 5255.8 & 2.50 & 0.53 \\
I & 3177.8 & 2529.4 & 5203.0 & 3.24 & 0.54 \\
II & 3224.0 & 3488.5 & 6718.9 & 2.29 & 0.23 \\
III & 400.0 & 411.0 & 391.0 & 18.80 & 4.00 \\
IV & 3368.4 & 5730.8 & - & 1.35 & - \\
\hline
\end{tabular}

$$
f_{\mathrm{sp} 1}\left(\bar{E}_{0}, t=\infty\right)=\mathbf{g}_{\mathrm{sp} 1}^{\mathrm{T}} \bar{E}_{0} \tilde{\mathbf{K}}_{\mathrm{re}} \mathbf{u}_{\mathrm{c}},
$$

where $\bar{E}_{0}$ is a guessed value of $E_{0}$.

Because $f_{\mathrm{sp} 1}\left(E_{0}, t=\infty\right)=a$, the following relationship holds

$$
\frac{1}{\bar{E}_{0}} f_{\mathrm{sp} 1}\left(\bar{E}_{0}, t=\infty\right)=\frac{1}{E_{0}} f_{\mathrm{sp} 1}\left(E_{0}, t=\infty\right)=\frac{a}{E_{0}} .
$$

$E_{0}$ can be identified by

$$
E_{0}=\frac{a \bar{E}_{0}}{f_{\mathrm{sp} 1}\left(\bar{E}_{0}, t=\infty\right)},
$$

where $f_{\mathrm{sp} 1}\left(\bar{E}_{0}, t=\infty\right)$ is obtained from the simulation result with a guess value $\bar{E}_{0}$ and $E_{i}=0, \tau_{i}=0$.

\subsubsection{Identification of $\boldsymbol{E}_{i}$ and $\tau_{i}$}

The other parameters of the generalized Maxwell model are identified by solving the optimization problem. The objective function is the squared error between the simulation and experimental results. The patternsearch () function of MATLAB Global Optimization Toolbox ${ }^{\mathrm{TM}}$ was chosen as the optimization problem solver that updates the parameters using pattern search algorithms.

As presented in Fig. 10, dynamic simulation is performed in every optimization loop. The solver sends updated parameters to the simulator. The simulator virtually reproduces the experiment, calculates the error between the simulation and experimental results, and sends the error back to the optimization solver. The simulator was programmed in the $\mathrm{C}++$ language. In the optimization problem, the simulator and MATLAB run in parallel 


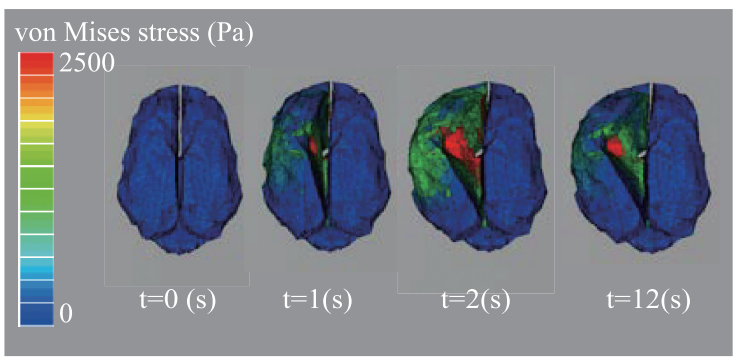

Fig. 11 Retraction simulation of $10 \mathrm{~mm} / \mathrm{s}$ with parameters of "II" in Table 1.

and bi-directionally communicate with each other.

The average force result of the experiments and experimental data of specimens No. 4 and No. 8 with a retraction velocity of $10 \mathrm{~mm} / \mathrm{s}$ were used as the reference $\left(f_{\text {fit }}(t)\right.$ in $\left.(33)\right)$ in the parameter identification. The initial guess was calculated by the parameters of the fitted curve presented in Section 4.2.1. The conditions of the optimization problem are listed as follows :

- Parameter vector :

$$
\mathbf{x}=\left[\begin{array}{ll}
E_{i} & \tau_{i}
\end{array}\right]^{\mathrm{T}}, \quad(1 \leq i \leq N)
$$

- Objective function :

Minimize $\Phi(\mathbf{x})$

$$
\Phi(\mathbf{x})=\sum_{t=0(\mathrm{~s})}^{t=12(\mathrm{~s})}\left(f_{\mathrm{fit}}(t)-f_{\mathrm{sim}}(t)\right)^{2}
$$

subject to

$$
E_{i} \in\left[10, \quad 10^{4}\right], \quad \tau_{i} \in\left[10^{-2}, 10\right]
$$

- Initial guess :

$$
\mathbf{x}_{0}=\left[b_{i} \times\left(E_{0} / a\right) \quad c_{i}\right]^{\mathrm{T}}
$$

where $E_{0}$ of the average experimental data, specimens No. 4, and No. 8 were identified to be $3224.0(\mathrm{~Pa})$, $2939.2(\mathrm{~Pa})$, and $3416.5(\mathrm{~Pa})$, respectively.

The identified parameters are listed in Table 1. In Table 1, "I" shows the averages of parameters identified for specimens No. 4 and No. 8. "II" shows the parameters identified using the average of experimental data of No. 4 and No. 8 with the five-element generalized Maxwell model $(N=2)$. "IV" shows the parameters identified using the average of experimental data of No. 4 and No. 8 with the three-element generalized Maxwell model $(N=1)$.

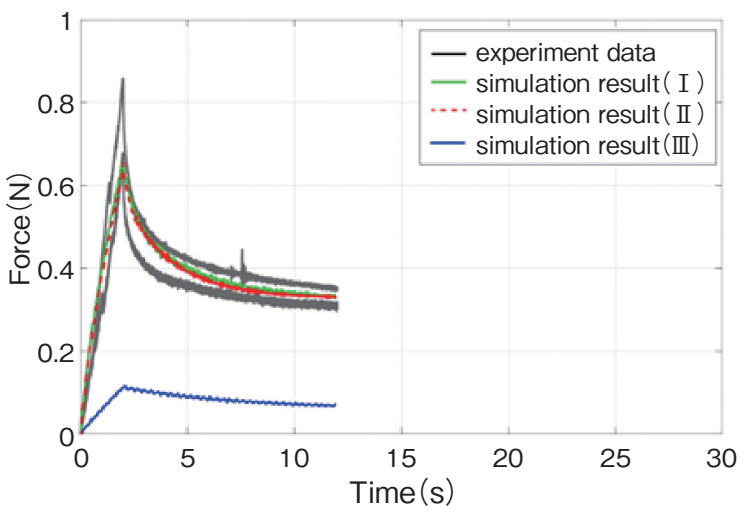

Fig. 12 Simulation results and experimental data with retraction velocity of $10 \mathrm{~mm} / \mathrm{s}$.

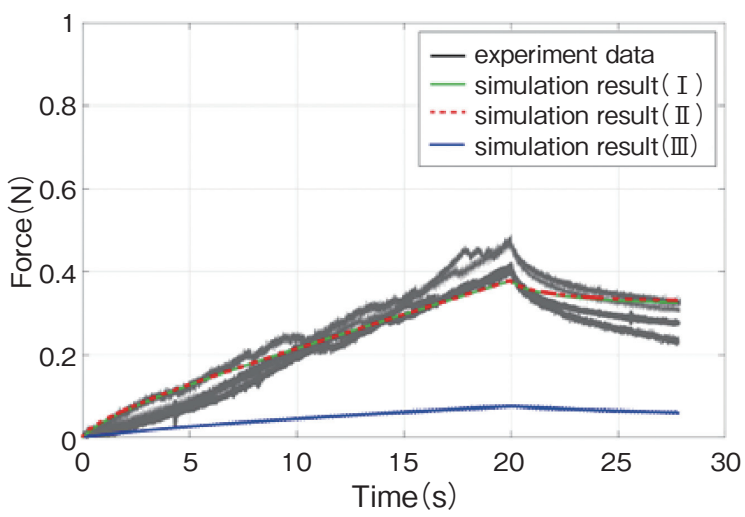

Fig. 13 Simulation results and experimental data with retraction velocity of $1 \mathrm{~mm} / \mathrm{s}$.

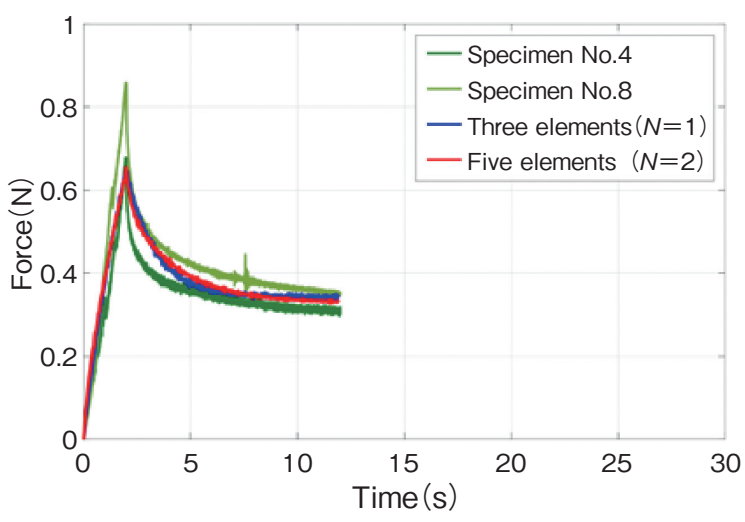

Fig. 14 Comparison of the number of elements of the generalized Maxwell model (retraction velocity : $10 \mathrm{~mm} / \mathrm{s}$ ).

\subsection{Simulation results}

Sequences of the simulation with a retraction velocity of $10 \mathrm{~mm} / \mathrm{s}$ using the parameters of " II" in Table 1 are shown in Fig. 11. Retraction simulations were conducted using the parameters listed as "I," “II," and "III" in Table 1 with a retraction velocity of $10 \mathrm{~mm} / \mathrm{s}$. The forces 
Table 2 Normalized mean square errors (NMSEs) between experimental data and simulation results. I : average of parameters identified for No. 4 and No. $8(N=2)$, II : parameters identified using the average of experimental results $(N=2)$, III : parameters identified in 21) $(N=2)$, and IV : parameters identified using the average of experimental results $(N=1)$.

\begin{tabular}{|c|c|c|c|c|c|}
\hline & & & $\mathrm{n} / \mathrm{s}$ & & $10 \mathrm{~mm} / \mathrm{s}$ \\
\hline & No. 1 & No. 5 & No. 10 & No. 11 & No. 4 No. 8 \\
\hline
\end{tabular}

\begin{tabular}{l|lllllll} 
NMSE ( I ) & 0.0125 & 0.0291 & 0.0242 & 0.0167 & 0.0202 & 0.0116
\end{tabular} \begin{tabular}{l|lllll|lll}
$\mathrm{NMSE}($ II $)$ & 0.0142 & 0.0284 & 0.0220 & 0.0160 & 0.0171 & 0.0063
\end{tabular} \begin{tabular}{l|llllllll}
$\operatorname{NMSE}($ III $)$ & 0.6738 & 0.6133 & 0.6163 & 0.6465 & 0.5965 & 0.6405
\end{tabular} \begin{tabular}{l|llllllll} 
NMSE (IV) & 0.0134 & 0.0345 & 0.0237 & 0.0146 & 0.0144 & 0.0096
\end{tabular}

Table 3 NMSEs between the average of experimental results plotted in Fig. 7 and each simulation result.

\begin{tabular}{l|c|c}
\hline & $1 \mathrm{~mm} / \mathrm{s}$ & $10 \mathrm{~mm} / \mathrm{s}$ \\
\hline $\operatorname{NMSE}$ ( I ) & 0.0114 & 0.0081 \\
$\operatorname{NMSE}$ (II) & 0.0109 & 0.0092 \\
$\operatorname{NMSE}$ (III) & 0.6377 & 0.6261 \\
$\operatorname{NMSE}$ (IV) & 0.0114 & 0.0051 \\
\hline
\end{tabular}

Table 4 NMSEs between the average of experimental results plotted in Fig. 7 and each experimental result.

\begin{tabular}{cccc|cc}
\hline \multicolumn{4}{c|}{$1 \mathrm{~mm} / \mathrm{s}$} & \multicolumn{2}{c}{$10 \mathrm{~mm} / \mathrm{s}$} \\
No. 1 & No. 5 & No. 10 & No. 11 & No. 4 & No. 8 \\
\hline 0.0214 & 0.0120 & 0.0093 & 0.0032 & 0.0112 & 0.0073 \\
\hline
\end{tabular}

$f_{\text {sp1 }}$ given by the simulations are plotted in Fig. 12 .

Simulations with a different retraction velocity $(1 \mathrm{~mm} /$ s) were also performed using the parameters listed in Table 1, which were identified using experimental results with a retraction velocity of $10 \mathrm{~mm} / \mathrm{s}$. The results are plotted in Fig. 13.

In 21), the parameters were identified by tensile tests using cylindrically clipped porcine brain specimens, the diameters of which were $5 \pm 1 \mathrm{~mm}$ and the heights of which were $10 \pm 1 \mathrm{~mm}$. The cylindrical specimens were stretched with a constant strain rate of 0.1 strain/s until the strain reached 0.1 , after which the length was held constant. The identified parameters are also listed in Table 1 "III" for comparison. As shown in Table 1, the parameters identified in 21) (simulation result (III)) are completely different from the parameters identified by the brain retraction experiments in this paper. As plotted in Fig. 12 and 13, simulations with the parameters identified in 21) could not reproduce the brain retraction experi- ments sufficiently well. It is considered that the cylindrical specimens used in 21) were exposed, whereas the whole brains used in this paper were covered by pia mater, and hence the whole brains were stiffer than the exposed brain parenchymas.

\section{Discussion}

\subsection{Accuracy of simulation results}

The normalized mean square error (NMSE) is used as an index of similarity between two datasets. In order to numerically evaluate the similarity between the simulation results and the experimental data, NMSEs were calculated. The smaller the NMSE, the higher the degree of similarity. The NMSE between the simulation and experimental results can be calculated as follows

$$
\mathrm{NSME}=\frac{1}{m} \sum_{i=1}^{m} \frac{\left(f_{\mathrm{sp} 1, \exp }(i)-f_{\mathrm{sp} 1, \mathrm{sim}}(i)\right)^{2}}{f_{\mathrm{sp} 1, \exp }^{2}(i)},
$$

where $f_{\text {sp1,exp }}$ is the force history of the experimental data, and $f_{\text {spl,sim }}$ is the reaction force history of the simulation. $m$ is the number of data. The NMSEs between the experimental data of each specimen and the simulation results with the "I," “II," “III," and "IV" parameters are described as NMSE ( I ) - (IV). The NMSEs between experimental datasets (Nos. 1, 5, 10, 11, 4, and 8) and simulation results ( I ) - (IV) are listed in Table 2. The NMSEs between the average of experimental results (Fig. 7) and simulation results ( I ) - (IV) are listed in Table 3.

The NMSEs between the experimental results and the simulation results with parameter set (III) (parameters identified in 21)) are apparently larger than those from other simulation results. The possible reason was discussed in Section 4.3. There are no significant differences between NMSEs with parameter set ( I ) and those with (II). It appears that the results with parameter set (II) are slightly better than those with ( I ).

The NMSEs between the average of the experimental results (Fig. 7) and each experimental result are listed in Table 4. The NMSEs listed in Table 4 denote the experimental deviations caused by the individuality of specimens, and their ranges are $[0.0032,0.0214](1 \mathrm{~mm} /$ s) and $[0.0073,0.0112](10 \mathrm{~mm} / \mathrm{s})$, whereas the ranges of NMSE between simulation results with parameter sets 
( I ), (II), and (IV), and the average of experimental results are $[0.0109,0.0114](1 \mathrm{~mm} / \mathrm{s})$ and $[0.0051$, $0.0092](10 \mathrm{~mm} / \mathrm{s}) \quad($ Table 3$)$. It is concluded that the errors of simulation results with parameter sets ( I ), ( II ), and (IV) are almost same with or less than the experimental deviations caused by the individuality of specimens.

\subsection{Number of elements of generalized Maxwell model}

In order to investigate the relationship between the number of elements of the generalized Maxwell model and the accuracy of the simulations, the three-element generalized Maxwell model $(N=1)$ was introduced in the simulation. The parameters were identified using the average of the experimental results with No. 4 and No. 8 in the same way as for (II), and are listed in Table 1 (IV). Simulation results with a retraction velocity of $10 \mathrm{~mm} / \mathrm{s}$ using the three-element and five-element generalized Maxwell model are shown in Fig. 14. The NMSEs of the three-element generalized Maxwell model are listed in Table 2 NMSE (IV). As seen in Table 2, there are no significant differences between NMSE (II) and (IV). The conclusion is that the number of the generalized Maxwell model elements does not significantly affect the accuracy of the brain retraction simulation.

\subsection{Computational time of simulation}

There are 5,212 nodes and 25,102 tetrahedral elements in the brain model. Numerical calculation is performed on a workstation KRONOS S810R (CIARA Inc., Intel Core i7 3960X, 6 cores, $3.3 \mathrm{GHz}$ ). A GPU (NVIDIA ${ }^{\circledR}$ Quadro 4000, 256 CUDA cores) was used to solve $\mathbf{u}_{\mathrm{f}}$ in (24) by using the conjugate gradient $(\mathrm{CG})$ method instead of matrix inversion.

The mean computational time of the simulation is 598 $\mathrm{ms} /$ loop. The computational time should be reduced for real-time simulation.

\subsection{Clinical outcome}

As shown in Fig. 12 and 13, although the parameters were identified with the experimental data of $10 \mathrm{~mm} / \mathrm{s}$, the experimental data of $1 \mathrm{~mm} / \mathrm{s}$ were well reproduced by the simulation (see Table 2). This proves the usefulness of the proposed model for brain retraction. The stress-time data of the brain retraction can be collected within the neurosurgeries ${ }^{11)}$. The parameters of the human brain can be identified. Therefore, the proposed model can be used in the neurosurgery simulations. The safety range of the retraction stress is reported in 11), 22). The simulation can evaluate whether the stress conditions are safe or not.

It is expected that the brain retraction simulator will be applied to intraoperative estimation of retraction stress in future to generate excessive stress alerts.

\section{Conclusion}

In this paper, brain retraction experiments with two retraction velocities were conducted using porcine brains. The mechanical characteristics were confirmed by experimental results. A considerable number of tensile and compression experiments using small pieces of animal brains have been reported ; however, the dynamic behavior of brain retraction for different retraction velocities has not been investigated very well. A numerical calculation method for viscoelasticity that is based on the generalized Maxwell model but considering the inertial term was proposed. The main advantage of the proposed model is that computational stability is guaranteed even when the stiffness matrix becomes singular. The parameters of the proposed viscoelastic model were identified by solving the optimization problem. Brain retraction simulations were performed using a $3 \mathrm{D}$ porcine brain $\mathrm{FE}$ model. A comparison of the simulation and experimental results shows that the simulation reproduces the mechanical characteristics of brain retraction well.

\section{Acknowledgements}

This work was supported by a JSPS Grant-in-Aid for JSPS Fellows (15J01496), a JSPS Grant-in-Aid for Scientific Research (A) (15H01707), and the JSPS funding program for next-generation world-leading researchers (LR003).

\section{References}

1) Aggarwal R, Black SA, Hance JR, et al. Virtual reality simulation training can improve inexperienced surgeons' endovascular skills. Eur J Vasc Endovasc Surg 2006;31:588593. 
2) Das P, Goyal T, Xue A, et al. Simulation training in neurological surgery. Austin Journal of Neurosurgery 2014;1:1004.

3) Luciano C, Banerjee $\mathrm{P}$, Florea L, et al. Design of the ImmersiveTouch ${ }^{\mathrm{TM}}$ : a high-performance haptic augmented virtual reality system. In 11 th International conference on human-computer interaction, Las Vegas, NV, 2005.

4) Delorme S, Laroche D, DiRaddo R, et al. Neurotouch: a physicsbased virtual simulator for cranial microneurosurgery training. Neurosurgery 2012;71:ons32-ons42.

5) Alaraj A, Charbel FT, Birk D, et al. Role of cranial and spinal virtual and augmented reality simulation using immersive touch modules in neurosurgical training. Neurosurgery 2013;72:115123.

6) Gélinas-Phaneuf $\mathrm{N}$, Choudhury $\mathrm{N}, \mathrm{Al}-\mathrm{Habib} \mathrm{AR}$, et al. Assessing performance in brain tumor resection using a novel virtual reality simulator. Int J Comput Assist Radiol Surg 2014; 9:1-9.

7) Andrews RJ, Bringas JR. A review of brain retraction and recommendations for minimizing intraoperative brain injury. Neurosurgery 1993;33:1052-1064.

8) Kivisaari RP, Salonen O, Ohman J. Basal brain injury in aneurysm surgery. Neurosurgery 2000;46:1070-1076.

9) Zhong J, Dujovny M, Perlin AR, et al. Brain retraction injury. Neurological research 2003;25:831-838.

10) Dean BL, Wallace RC, Zabramski JM, et al. Incidence of superficial sylvian vein compromise and postoperative effects on CT imaging after surgical clipping of middle cerebral artery aneurysms. AJNR Am Neuroradiol 2005;26:2019-2026.

11) Rosenørn J. Self-retaining brain retractor pressure during intracranial procedures. Acta Neurochir (Wien) 1987;85:17-22.

12) Prevost TP, Balakrishnan A, Suresh S, et al. Biomechanics of brain tissue. Acta Biomater 2011;7:83-95.

13) Budday S, Nay R, de Rooij R, et al. Mechanical properties of gray and white matter brain tissue by indentation. J Mech Behav Biomed Mater 2015;46:318-330.

14) Wiechert E. Ueber elastische nachwirkung. Ph. D. dissertation, Hartungsche Buchdr., 1889.

15) Elkin BS, Ilankova A, Morrison B. Dynamic, regional mechanical properties of the porcine brain: indentation in the coronal plane. J Biomech Eng 2011;133:071009.

16) Kaliske M, Rothert H. Formulation and implementation of threedimensional viscoelasticity at small and finite strains. Computational Mechanics 1997;19:228-239.

17) Sedef M, Samur E, Basdogan C. Real-time finite-element simulation of linear viscoelastic tissue behavior based on experimental data. IEEE Comput Graph Appl 2006;26:58-68.
18) Newmark NM. A method of computation for structural dynamics. in Proc. ASCE 1959;85:67-94.

19) Saikali S, Meurice P, Sauleau P, et al. A three-dimensional digital segmented and deformable brain atlas of the domestic pig. J Neurosci Method 2010;192:102-109.

20) Digital atlas of the pig's brain. https://www6.rennes.inra.fr/adnc_eng/Downloads/Documenta tion.

21) Chen X, Sase K, Konno A, et al. A viscoelastic model of brain parenchyma for haptic brain surgery simulations. In 2016 IEEE/ SICE International Symposium on System Integration (SII2016) 2016:1656-1661.

22) Rosenørn J, Diemer N. The risk of cerebral damage during graded brain retractor pressure in the rat. J Biomech Eng 1985; 63:608-611 